\title{
Calibrating the elements of a multispectral imaging system.
}

\author{
Miguel A. López-Álvarez ${ }^{1}$, Javier Hernández-Andrés ${ }^{2}$, Javier Romero² ${ }^{2}$ Joaquín Campos $^{3}$ and
} Alicia Pons ${ }^{3}$.

${ }^{1}$ Large Format Printing Division, Hewlett-Packard Spain, 08174 Sant Cugat del Vallès, Barcelona, SPAIN.migangel@ugr.es

${ }^{2}$ Color Imaging Laboratory, Departamento de Óptica, Universidad de Granada, 18071 Granada, SPAIN.javierha@ugr.es, jromero@ugr.es

${ }^{3}$ Departamento de Metrología, Instituto de Física Aplicada, CSIC, 28006 Madrid, SPAIN. joaquin.campos@ifa.cetef.csic.es, alicia.pons@ifa.cetef.csic.es

We describe a method to calibrate the elements of a multispectral system aimed at skylight imaging, which consists of a monochrome charge-coupled device (CCD) camera and a liquid-crystal tunable filter (LCTF). We demonstrate how to calibrate these two devices in order to build a multispectral camera that can obtain spectroradiometric measurements of skylight. Spectral characterizations of the tunable filter and the camera are presented together with a complete study into correcting temporal and spatial noise, which is of key importance in CCDs. We describe all the necessary steps to undertake this work and all the additional instrumentation that must be used to calibrate the radiometric devices correctly. We show how this complete study of our multispectral system allows us to use it as an accurate, high resolution spectroradiometer.

Keywords: multispectral system; calibration; noise; CCD; LCTF; skylight; imaging. 


\section{Introduction}

Multispectral imaging systems and techniques ${ }^{1-6}$ have become powerful tools for the rapid measurement of high spatial resolution spectral images. They allow us to recover the spectral radiance of an illuminant, the reflectance of an object or the combined color signal ${ }^{7}$ at every pixel of the image by using data from the responses of only a few sensors. The spectral responsiveness of these sensors can be obtained by two different procedures. First, a trichromatic digital CCD camera with one or more broadband color filters attached to it can be used to take an image of the scene with each filter ${ }^{8-10}$. This technique is fast, easy to implement and provides good results in certain situations if the correct filters are chosen. Second, a monochrome CCD camera attached to a tunable filter (liquid crystal [LCTF] or acoustic-optic [AOTF] technologies are available) or a filter wheel can be used. Both options allow to a filter to be placed before the lens before taking each image from the scene $e^{1,6,11-15}$. With the latter approach the filters are usually narrow band and hence each image needs high exposure time, so noise must be taken adequately into account.

We built our multispectral imaging system by making use of a cooled 12-bit, monochrome, CCD camera (model Retiga QImaging SRV1394) and a Varispec LCTF (Cambridge Research Instruments). The camera has a spatial resolution of 1392x1040 pixels, with a pixel size of $6.45 \mu \mathrm{m} \mathrm{x}$ $6.45 \mu \mathrm{m}$, and its sensor is cooled to a constant $-30{ }^{\circ} \mathrm{C}$. The LCTF can be tuned from $400 \mathrm{~nm}$ to $720 \mathrm{~nm}$ in 10nm steps with full width at half maximum (FWHM) between $7 \mathrm{~nm}$ and $15 \mathrm{~nm}$, depending upon the central wavelength selected. These two devices can be used together to obtain accurate spectroradiometric measurements at every pixel of the imaged scene. We will demonstrate the accuracy of the results by comparing the measurements of our multispectral system with those obtained by using a spectroradiometer SpectraScan PR650. 
Scientific researchers in many fields may benefit from high resolution angular maps of the spectral power distribution (SPD) of skylight across the whole sky dome ${ }^{2,3,9,12,13}$. This information could be used, for instance, to estimate the experimental values of climate parameters ${ }^{9}$, or in algorithms for automatic cloud detection, among many other possible applications. Since the sky dome is the imaged object, the camera's lens is always focused to infinity, thus avoiding problems of chromatic aberration frequently experienced in systems using LCTFs.

Section 2 shows how we measure the spectral transmittance of the 33 selected channels of the LCTF, taking into account that liquid-crystal devices act as linear polarizers. In Sections 3 and 4 we focus on calibrating the CCD camera ${ }^{16,17}$. In Section 3 we conduct an exhaustive study into temporal and spatial noise generated by the CCD sensor array and how to remove as much of it as possible. By 'noise' we mean any process that reduces the ideal behavior of the camera's response with as far as time and radiant exposure changes ${ }^{16-24}$ are concerned. In Section 4 we measure the spectral response of our CCD camera so as to be able to take it into account when making spectroradiometric measurements. Finally, in Section 5 we describe a simple practical case involving skylight imaging where the measurements from the calibrated multispectral system are compared with the measurements from the PR650, thus demonstrating that our calibration process is accurate enough for spectral imaging purposes. We discuss our conclusions in Section 6.

\section{Spectral calibration of the LCTF.}

Before measuring the spectral transmittance of the modes (or peak wavelengths) of the LCTF, we must remember that this device, as all liquid crystal devices, has a linear polarizer at its input. Thus, if we are only interested in measuring its spectral transmittance we must assure that light impinges on it being linearly polarized, with its plane of vibration matching the direction described by the polarization 
transmission line of the LCTF. Otherwise we would perceive intensity attenuation due to absorption by the polarizer rather than by the filter per se. For this reason we also used a linear polarizer to obtain the adequate light polarization state impinging on the LCTF. We made sure in all the experiments that the transmission lines of the polarizer and the LCTF were perfectly aligned (the direction of both transmission lines were calculated in our laboratory).

The spectral calibration of the LCTF also involves two additional problems. First, the transmittance of the LCTF is quite low (particularly when tuned in short-wavelength modes) and so we must use a strong enough light source to ensure that the measurements needed to calculate the transmittance curves are affected as little as possible by noise. Second, the light source used to measure the transmittance of the LCTF must contain sufficient power throughout the spectral range in question (from $400 \mathrm{~nm}$ to $720 \mathrm{~nm}$ ). Some authors ${ }^{25}$ have succeeded in doing this by using an integrating sphere and tunable lasers, instrumentation which is not easy to come by. To overcome these two problems we propose to use skylight on completely clear days as an intense, spatio-temporal, homogeneous light source $^{26}$ (at least in the field of view of our instruments and within the short time needed for our measurements). Skylight is, nevertheless, a partially polarized light source, and this is an issue that should also be born in mind, as we explain later in more detail.

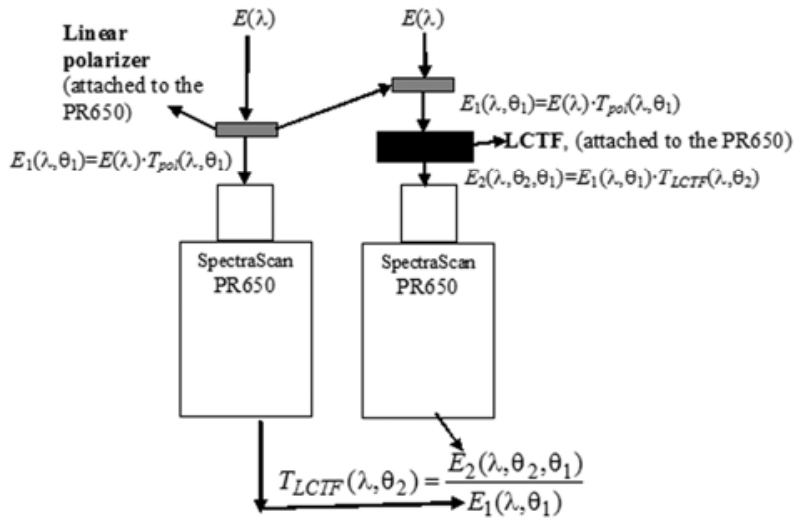


Figure 1. Diagram of the setup for measuring the spectral transmittance of the LCTF modes.

Figure 1 shows a scheme of the setup we followed for measuring the spectral transmittance of the LCTF modes. We used the PR650 spectroradiometer with a linear polarizer attached to it pointing at the sky (preferably towards the zenith) on a completely clear day. For the first measurement we obtained $E_{1}\left(\lambda, \theta_{1}\right)$. No more than five seconds later we made a second measurement after inserting the LCTF (tuned to the corresponding mode or central wavelength) between the polarizer and the PR650 (always making sure that the polarization directions of the polarizer and the LCTF were perfectly aligned) and obtained $E_{2}\left(\lambda, \theta_{1}, \theta_{2}\right)$. Since the polarizer is perfectly aligned with the LCTF, angles $\theta_{1}$ and $\theta_{2}$ are identical and hence $E_{2}$ depends upon $\theta_{1}$ alone. In Figure 1 the magnitude we are interested in measuring is $T_{L C T F}(\lambda)$, which is the spectral transmittance of the selected mode on the LCTF. $T_{\text {pol }}(\lambda)$ is the spectral transmittance of the polarizer and $E(\lambda)$ is the radiance coming from the zenith.

By calculating the ratio between $E_{2}$ and $E_{1}$ we can obtain $T_{L C T F}(\lambda)$ in the selected mode of the LCTF. Following this procedure we can measure the spectral transmittance of the LCTF when linearly polarized light impinges on it. We repeated each measurement three times from $400 \mathrm{~nm}$ to $720 \mathrm{~nm}$, tuning the filter to the selected modes. Figure 2 shows the curves thus obtained with error bars corresponding to the standard deviation achieved throughout the three series of measurements. These curves obtained using our method are very close to the information provided by the manufacturer and also to the curves measured by other authors with an identical device 27,28 . 


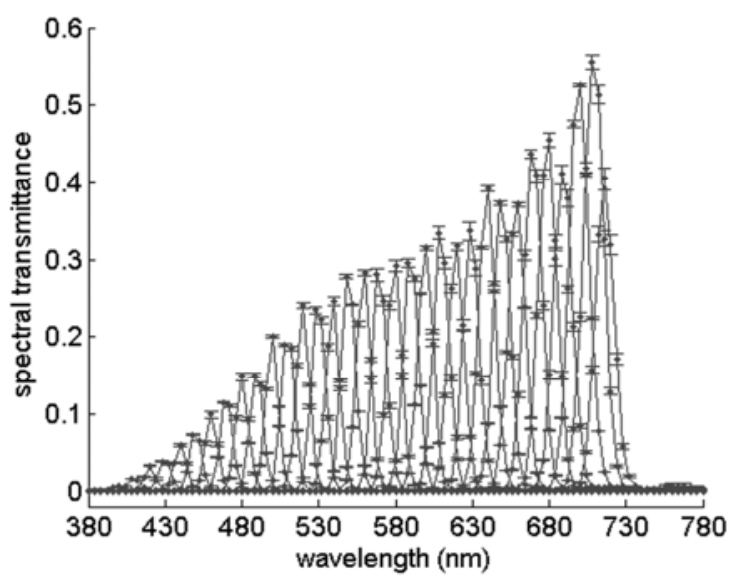

Figure 2. Spectral transmittance of the 33 modes of the Varispec liquid-crystal tunable filter measured in our laboratory (error bars show the standard deviation obtained at each wavelength sampled).

\section{3.- Correction of the CCD camera's response.}

In this section we detail the entire process we propose to calibrate radiometrically a monochrome CCD camera $^{16-20,22}$, which mainly involves two steps: studying and correcting the influence of different kinds of noise, and studying the spectral response of the CCD sensor. In the following subsections we show how we developed the necessary steps to accomplish the complete calibration of our camera, and the instrumentation used in each case.

Several publications exist describing the types of noise and their influence on CCD camera response $e^{5,8,13,16-24,29}$. Detailing the effect of noise on electronic devices is outside the scope of this paper but it is important to consider the nature of the major noise sources in order to understand how to minimize them.

Shot noise is the noise associated with the random arrival of photons at the CCD and it grows proportionally to $\mathrm{Q}^{1 / 2}$, $\mathrm{Q}$ being the total charge collected in a pixel (as governed by Poisson's statistics $)^{16,29}$. In addition, some of the electrons within each pixel of the CCD are generated thermally and this is also affected by noise (dark response). The number of these thermal electrons in a pixel is proportional to temperature and exposure time. All excited electrons suffer from generation- 
recombination noise. Furthermore, thermal, or Johnson, noise is produced as the stored electrons move along the CCD and associated electronics. Flicker or 1/f noise is associated with the output amplifier attached to every CCD matrix sensor, and it is generally accepted that flicker noise is generated by the tunneling of electrons into the oxide and by surface states which generate energy levels within the forbidden gap $^{29}$, hence trapping electrons that are released later and disturbing the total current. This type of noise varies inversely with the frequency of variation of the currents inside the device, which in CCD devices is related to the readout frequency. Finally, quantization noise, another important noise source, is due to the round effects present in any A/D conversion.

Bearing in mind the nature of noise, we may conclude that cooling down the CCD will reduce noise as well as the dark counts. To this end we cooled our CCD down to $-30{ }^{\circ} \mathrm{C}$. Nevertheless, from a radiometric point of view, it is more interesting to study high- and low-frequency temporal noise from an overall point of view, since high-frequency temporal noise can be minimized by averaging frames, while low-frequency noise will be the limiting noise measurement.

\section{1.- Estimating high-frequency temporal noise.}

High-frequency temporal noise refers to that noise which varies fast enough to be detected in periods of under one minute ${ }^{18}$. This undesired noise can easily be removed if we take and average a set of consecutive frames. Hence, the aim of this experiment was to find the minimum number of frames that must be averaged to minimize the effect of high-frequency noise. We also wanted to ascertain whether this minimum number of averaged frames depends upon exposure time.

The experimental setup is shown in Figure 3. We had an integrating sphere $50 \mathrm{~cm}$ in diameter with an input and an output port in order to have a large and uniform source to be imaged by the camera. Polychromatic light from a $420 \mathrm{~W}$ incandescent lamp (current stabilized to better than $1 \mathrm{~mA}$ ) 
was used. The output port was set at $90^{\circ}$ from the input port and the CCD camera was placed right in front of it on top of a rail, perfectly aligned so that the reading from the CCD was optimized.

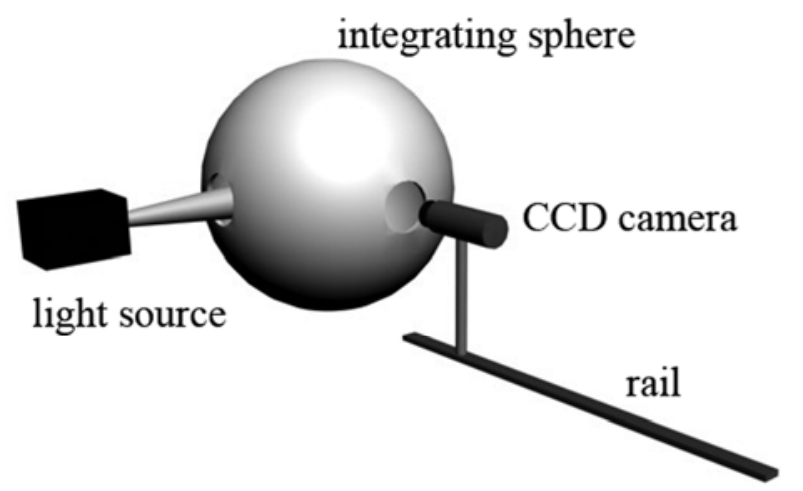

Figure 3. Experimental setup for measuring high-frequency temporal noise.

For each exposure time we placed the light source at such a distance from the input port that the camera registered near to the maximum number of digital counts without reaching saturation. We used exposure times of 7, 16, 95 and 1000 ms, considering this to be a reasonable range. Since our camera is 12-bit, the maximum corresponds to 4095 digital counts. Thus in every frame we took with our camera we tried to get no more than 3500 digital counts (about $85 \%$ of the maximum) in any pixel. Some authors $^{16,17,22}$ recommend not exceeding a limit of $90 \%$ in order to avoid saturation and work safely in the linear region of the CCD sensor. By assuring a constant number of registered digital counts and a constant temperature of the sensor (thanks to the cooling system of the camera) we avoided the influence of both parameters on camera noise ${ }^{17}$.

We took series of $p=20,80150$, and 300 frames to study the influence of the number of averaged frames on the minimization of high-frequency temporal noise, which was measured by calculating the residual spatial standard deviation across the whole CCD sensor array. The smaller this residual spatial standard deviation is, the more temporal noise is removed in the averaging process. In Figure 4 we show the residual spatial standard deviation versus the number of averaged frames, $p$, for 
the different exposure times. It can be seen how an increase in the exposure time results in a concomitant increase in residual noise in the camera since the flicker or $1 / \mathrm{f}$ noise ${ }^{16,23}$ rises with exposure time, whilst thermal noise and shot noise depend only on temperature and the number of digital counts ${ }^{24}$ respectively. In our experiment, for exposure times under $100 \mathrm{~ms}$ the residual spatial noise was almost of the same magnitude, whilst it was considerably higher for an exposure time of 1000 ms. This was due to the influence of flicker noise when the exposure time was long, since thermal and shot noise are considered to be 'white noise', i.e. they are equally intense for every exposure time. This experiment proves that we can compensate for flicker noise if we operate with small exposure times but cannot avoid the 'plateau' value imposed by 'white noise' sources such as thermal and shot noise.

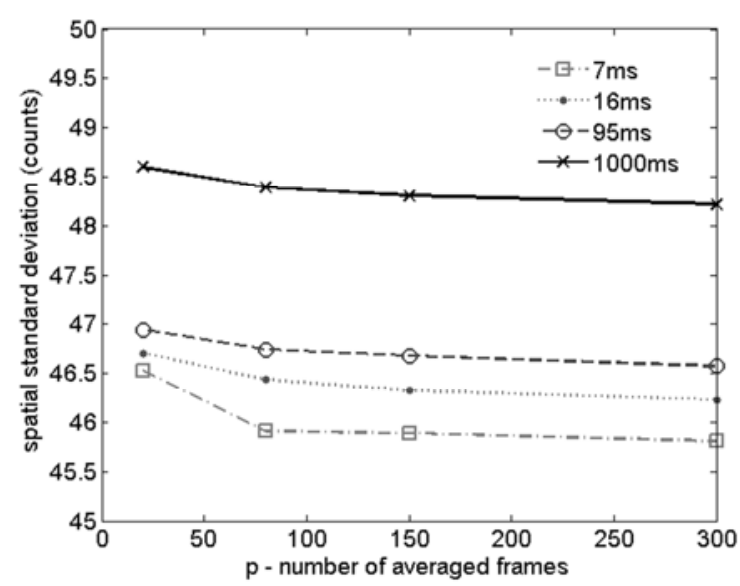

Figure 4. Residual spatial standard deviation for different exposure times as a function of averaged frames.

It can also be seen in Figure 4 how noise is barely reduced when we average more than 100 frames approximately. This number seems to be independent of the exposure time and hence we can think of it as a CCD camera figure. 


\section{2.- Estimating low-frequency temporal noise.}

In this section we measure the kind of noise that affects the camera when it is used for long periods of time (more than one minute at least ${ }^{18}$ ). By this we do not mean that the exposure time is longer than one minute, but that the response of the camera can be affected for several reasons, causing it to change slightly through time. These reasons are known as low frequency noise ${ }^{18}$. Moreover, it is important to make sure that the spatial average of the pixels in the CCD array and all the individual pixels behave similarly over long periods of time because, if their responses do not vary together, the algorithms used for the spatial correction of the camera response (shown below) would not be valid, since this correction would not be constant in time. The experiment shown in this section allows us to estimate low-frequency spatial noise and at the same time to find any individual pixels behaving abnormally ${ }^{18}$.

We took series of 100 frames each with a fixed exposure time of $10 \mathrm{~ms}$, every five minutes for three hours (36 series of images finally), recording only the dark response of the camera (in the absence of any light source). By taking only the dark response of the camera we avoid the influence of highfrequency temporal noise (mainly shot noise, which increases concomitantly with the digital counts registered with the camera). For each of the 36 series obtained in this way we can calculate the temporal average over the 100 frames of the difference between each individual pixel value, $C_{i}$, and the

spatial average across the whole CCD array, $\left\langle C>\right.$. This magnitude is then $\overline{C_{i}-<C>}$, the upper bar indicating the temporal average. We can also calculate the standard deviation, $\sigma\left(\overline{C_{i}-<C>}\right)$, throughout the 36 series, which gives us an estimation of the temporal variation of the response of each individual pixel compared to the spatial average across the whole CCD sensor array. In other words, it shows whether the response of each individual pixel varies together with the spatial average response. 


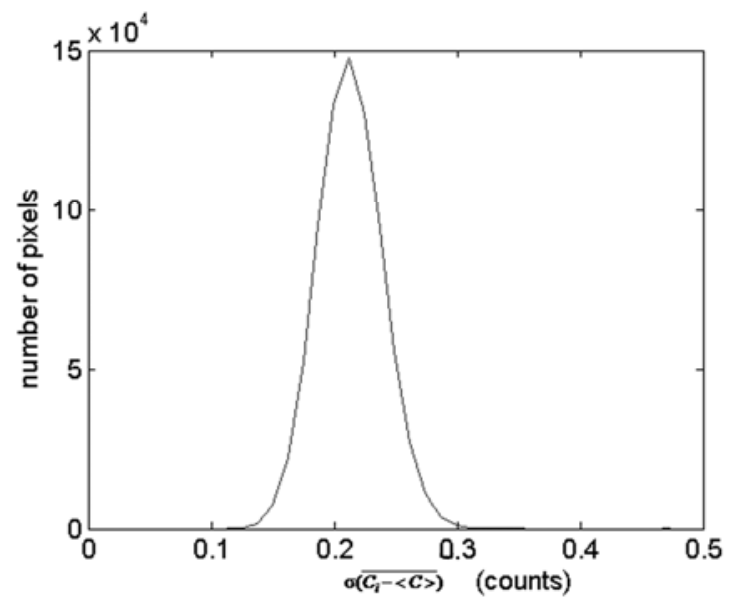

Figure 5. Histogram showing the standard deviation of the difference between individual pixel response and the frame's average response, $\sigma\left(\overline{C_{i}-<C>}\right)$, for our CCD camera.

Figure 5 shows a histogram with the number of pixels having a certain value of the standard deviation $\sigma\left(\overline{C_{i}-<C>}\right)$. Some authors ${ }^{18}$ have found in similar experiments that some CCD sensors show various peaks in the histogram, revealing the existence of abnormal pixels in the CCD array. In our case, however, every pixel of the CCD camera varied together with the response of the spatial average. We can see that our histogram is centered on a value of 0.22 digital counts, which theoretically corresponds ${ }^{18}$ to the readout noise magnitude ${ }^{16}$ of 2.2 digital counts provided by the manufacturer $^{30}$.

\subsection{Reciprocity law.}

Several authors ${ }^{1-6,8,16-22}$ put forward the idea of modelling the pixel response of CCD cameras linearly, i.e.

$$
C_{i}=C_{o, i}+R_{i} E_{i} t_{\exp }
$$


where the digital counts on pixel i, $C_{i}$, relates linearly to the exposure time, $t_{\text {exp }}$, the irradiance, $E_{i}$, and the responsiveness of that pixel, $R_{i}$. The additive term, $C_{o, i}$, represents the dark current response that always affects any electronic imaging device or detector, and can be estimated in the absence of light. If we assume that the pixel's responsiveness, $R_{i}$, is constant, then we will obtain the same response, $C_{i}$, while the product $E_{i} t_{\text {exp }}$ is constant. For example, we could double the exposure time while closing the aperture so that the received irradiance is halved, and the response of the camera would be the same. This behavior is known as 'reciprocity law, ${ }^{, 17}$. Nevertheless, it is possible that this law is not fulfilled under some circumstances, and we focus on this possibility in this section: to study the dependence of $R_{i}$ upon $t_{\text {exp }}$ in order to find some ranges where the reciprocity law does not hold good.

Using the same experimental setup as that shown in Figure 3, we took series of 100 frames of the integrating sphere output port and their corresponding dark frames, with exposure times ranging from $10 \mu \mathrm{s}$ up to $100 \mathrm{~ms}$. We calculated the ratio $\frac{\overline{C_{i}-C_{o, i}}}{t_{\exp }}$ for every CCD pixel, eliminating the influence of high-frequency temporal noise with the average of the 100 frames, as explained in Section 3.1. In Equation (1) we can see that this magnitude corresponds to the product of responsiveness, $R_{i}$, and irradiance, $E_{i}$, which was not measured in this experiment, but was constant throughout the procedure. In this way we can calculate the relative value of responsiveness compared, for example, to its value at $100 \mathrm{~ms}$ exposure time, and then check whether this responsiveness depends upon $t_{\text {exp }}$. This is enough to check whether the reciprocity law holds good for our CCD camera.

Figure 6 shows the spatial average of relative responsiveness across the CCD array, $\left\langle R^{p}\right\rangle$, against the exposure time used in this experiment. We can see that for exposure times under $1 \mathrm{~ms}$ responsiveness greatly depends upon exposure time and hence the reciprocity law is not valid in that region. For exposure times of more than $4 \mathrm{~ms}$ the variation in responsiveness is below 2\% (limit 
represented by horizontal lines in Figure 6), which is below responsiveness uncertainty and thus it may be considered that the reciprocity law is fulfilled. This behavior is known as "smear" and has to be taken into account when using cameras with under 4 ms exposure times.

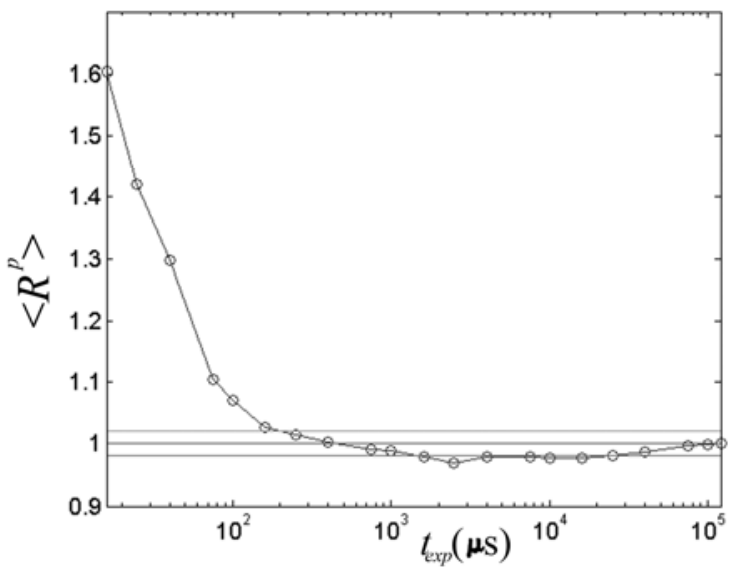

Figure 6. Responsiveness spatial average relative to its value at $100 \mathrm{~ms}$ versus exposure time.

\subsection{Non-linearity and spatial non-uniformity:}

Pixel responsiveness in a CCD, $R_{i}$, not only depends upon exposure time, as we explained in the previous section, but it also varies with the number of digital counts registered at that pixel ${ }^{19}$, even if we are in the situation where the reciprocity law holds good. From Equation (1), ideal responsiveness would be

$$
R_{i}=\frac{C_{i}-C_{o, i}}{E_{i} t_{\exp }}
$$

If we want to take into account the non-linear effect we are referring to here, we must introduce a factor into Equation (2) that embraces this variation:

$$
R_{i}^{0}(\lambda)=\frac{C_{i}-C_{o, i}}{E_{i} t_{\exp } r^{N L}(C)}
$$

The new factor, $r^{N L}$, depends upon the digital counts $C=C_{i}-C_{o, i}$ registered at the camera. 
There are still other effects, such as spatial non-uniformity in the sensor array, that influence the response of the camera. We can also take this effect into account by modifying the expression of the responsiveness:

$$
R_{i}(\lambda)=R_{i}^{0}(\lambda) r^{L N}(C)=\left\langle R_{i}^{0}(\lambda)\right\rangle r^{N U} r^{N L}(C)
$$

where we have explicitly separated the variation in responsiveness due to non-linear effects, $r^{N L}$, and spatial non-uniformity, $r^{N U}$, since the spatial average $\left\langle R_{i}^{0}(\lambda)\right.$ is constant. The dependence upon wavelength (or spectral calibration) will be studied in the following section once we have estimated these two correction parameters.

The factor correcting non-linearity can be calculated in a straightforward experiment, once more using the experimental setup in Figure 3. We will use exposure times over 4 ms to be sure that the reciprocity law is fulfilled. The light source was placed in such a way that for an exposure time of 4 ms we got about 500 digital counts on the camera, and for $100 \mathrm{~ms}$ the reading was around 3000 digital counts (after removing the dark current noise and averaging 100 frames). Figure 7 shows the values for the average non-linearity correction factor, $r^{N L}$, across the CCD array for various digital count levels, $C$, registered by the camera. We can see how this factor decreases considerably below 2500 digital counts, proving that it is necessary to take it into account in order to correct this effect. Figure 7 also shows the error bars corresponding to the spatial standard deviation of this correction parameter across the CCD array. We have also plotted the best second-degree polynomial that fits the experimental points, which will be used as a theoretical function for correcting non-linearity in further measurements taken with our camera.

After correcting the camera response from non-linearity, we might now expect its behavior to be much closer to the ideal. Figure 8 shows the spatial average of responsiveness $\left\langle R_{i}^{0}(\lambda)\right.$ (normalized 
to its value at $10 \mathrm{~ms}$ ) after using the correction factor $r^{N L}$ (and normalized to its value at $10 \mathrm{~ms}$ ). The remaining variability is lower than $0.3 \%$ and is completely random (i.e. it does not depend on the number of digital counts). By doing this we have extended the counts range within which reciprocity law holds good from 500 to 3000 digital counts.

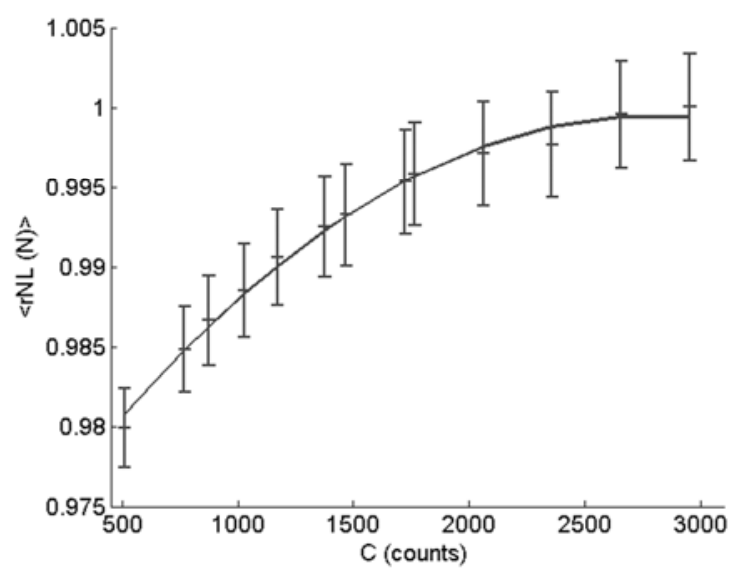

Figure 7. Non-linearity correction factor as a function of the registered digital counts.

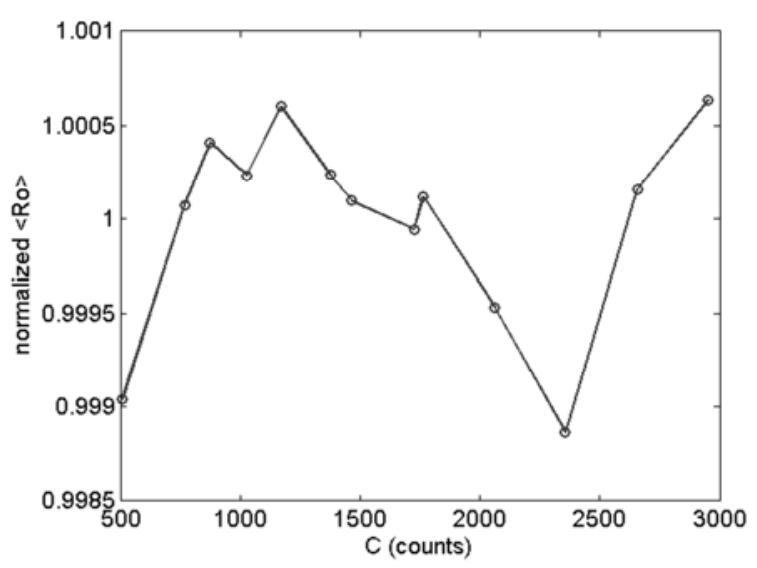

Figure 8. Spatial average of responsiveness corrected for non-linearity and normalized to its value at 10 ms, as a function of the digital counts registered by the camera. 
Finally, we need only to correct for spatial non-uniformity, as we mentioned in Equation (4), in which we introduced the $r^{N U}$ correction factor. The sources of spatial non-uniformity in CCDs are manifold ${ }^{22}$, but fortunately they are almost constant versus time and thus can be adequately corrected. From Equations (1) to (4) we can easily obtain ${ }^{19}$

$$
r_{i}^{N U}=\frac{C_{i}-C_{o, i}}{r_{i}^{N L}(C)<R_{i}^{0}(\lambda)>E(\lambda) t_{\exp }}
$$

The main advantage of this method is that we can separate the effects of wavelength dependence and spatial non-uniformity from other factors that exert major influence on camera response. Other authors ${ }^{16}$ have proposed the use of so-called "flat-field correction algorithms" where such separation is not so clear, and hence their spatial corrections depended on the spectral power distribution of the illuminant used ${ }^{18}$.

We calculated $r_{i}^{N U}$ for each camera pixel, using the same set of images mentioned in this section and applying Equation (5). Figure 9a shows the resulting spatial correction array, i.e. the value of $r_{i}^{N U}$ at every pixel of the CCD array sensor, which must be used in the denominator of Equation (6) to correct the sensor data (the origin of this equation is discussed later). We can see how small corrections are applied in the entire CCD matrix, and a value below 1 is used near the corners of the image in order to increase the value registered in those pixels. This is a typical effect caused by any camera lens. Figure 9b shows the effect of applying this correction factor to an image of the integrating sphere port, and it can be seen how the standard deviation across the CCD array decreases from 3\% to $0.27 \%$ at 3000 digital counts, thus proving that the inclusion of the correction factor for spatial nonuniformity really improves the homogeneity of the CCD array response. Figure 10 shows the value of the pixels in an image of the integrating sphere before and after applying the spatial non-uniformity correction. We should notice that this image was taken with a different light source from the one used 
to calculate the correction parameters, thus proving that our method depends in no way upon the spectral-power distribution of the incident irradiance.

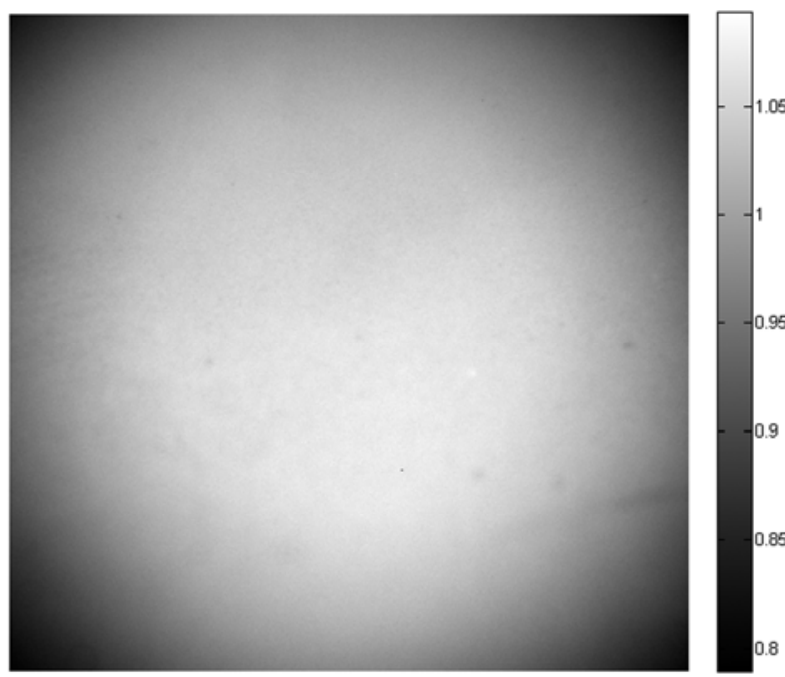

(a)

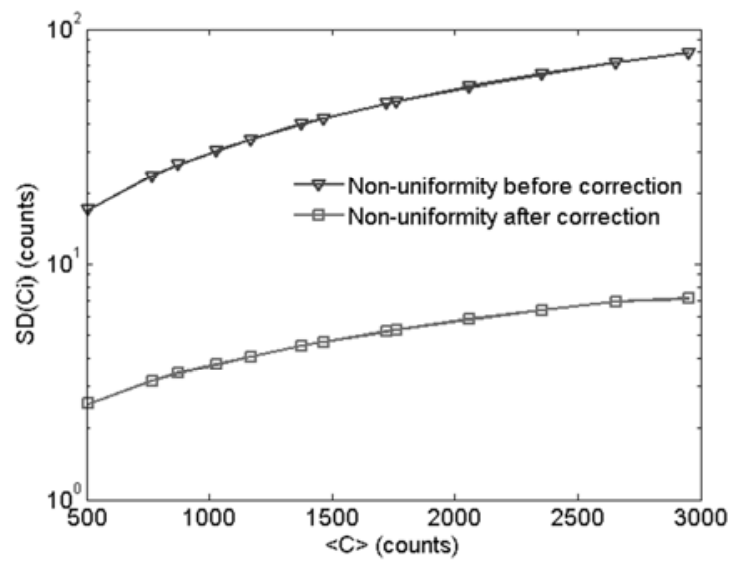

(b)

Figure 9. a) Spatial correction array; b) Improvement in spatial non-uniformity. 

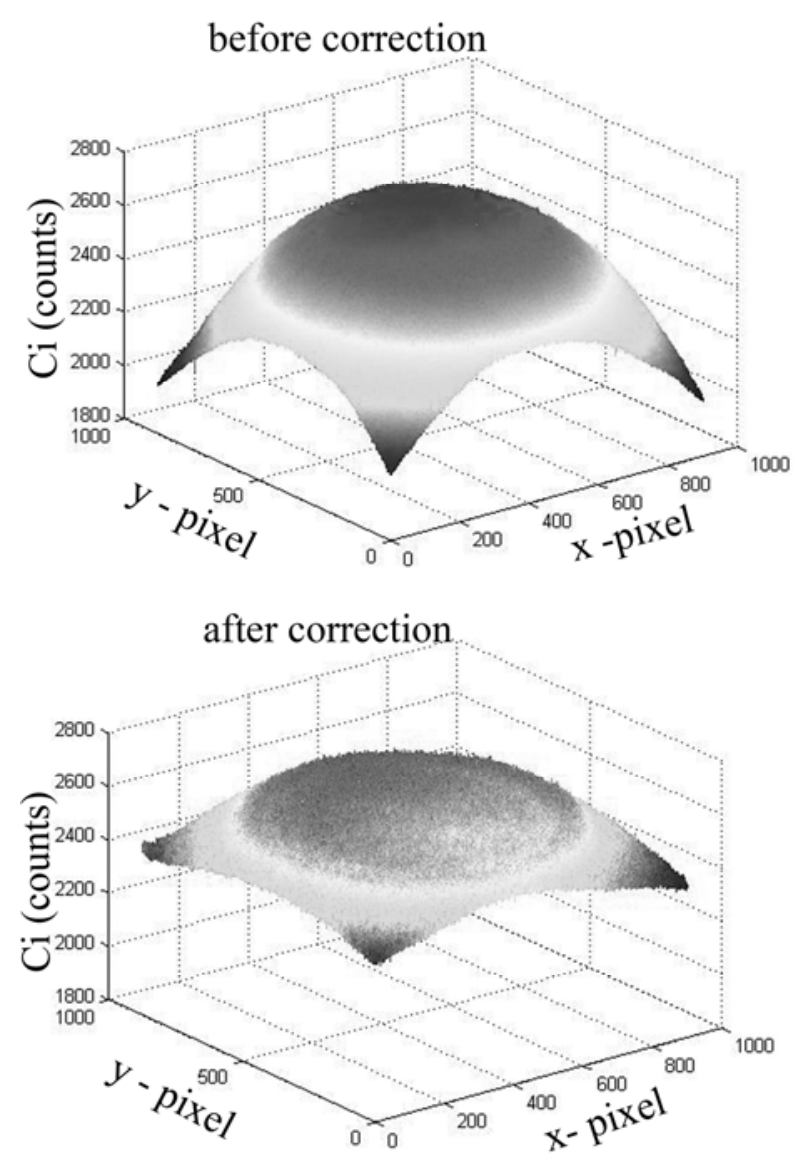

Figure 10. Effect of the spatial non-uniformity correction factor on an image of an integrating sphere output port.

We can summarize this section by pointing out that the corrected pixel value, $C_{i}^{c}$, after applying the corrections studied here can be calculated as

$$
C_{i}^{C}=\frac{C_{i}-C_{o, i}}{r_{i}^{L N}(C) r_{i}^{N U}(\lambda)}
$$

These pixel values are corrected for temporal noise, CCD non-linearity and spatial non-uniformity. They also comply with the reciprocity law and so, once we have performed the spectral calibration of the CCD, they can be used to make the camera work as a precise radiometric instrument by means of Equation (7): 


$$
E_{i}(\lambda)=\frac{C_{i}^{c}}{R_{i}^{0}(\lambda) t_{\exp }}
$$

\section{Spectral calibration of the camera.}

Spectral calibration of CCD devices is a frequently studied subject, involving two main approaches. By using a monochromatic light source (from a monochromator or a tunable laser) and an integrating sphere it is possible to measure the responsiveness of the CCD for a certain wavelength and then cover

all the visible range by tuning the wavelength of the light source ${ }^{11,17}$. Otherwise, a set of known spectra from the patches of a color target may be related to the responses of the camera to those spectra in order to estimate the spectral responsiveness of their sensors ${ }^{1,31}$. Really accurate results can be obtained by following this indirect procedure.

Our proposal is closer to the methods described by the former authors, since we try to use a monochromatic light source for each wavelength at which we want to discover the spectral responsiveness of the camera. Nevertheless, instead of using a sophisticated monochromator or a tunable laser we use our LCTF as an easy-to-use monochromator to obtain the responsiveness of the camera at the central wavelength of each of the filter modes. Furthermore, instead of using an integrating sphere as a perfectly homogeneous object, we take images of the zenith on perfectly clear days for this purpose, as we did in Section 2. We do this because we only have access to tunable lasers that can cover a small portion of the visible range and the low transmittance of the LCTF modes forces us to use a very intense light source. Nevertheless, we will compare the results of our method against the latter approach using tunable lasers in the spectral regions where they overlap.

When developing our method for spectral calibration of the camera, we made two assumptions. First, each of the transmittance modes of the LCTF is narrow enough to assume that the radiance information received by the camera when a filter mode is tuned corresponds to the central wavelength 
alone (i.e. we assume that the modes of the LCTF are equivalent to monochromatic filters). Since the typical spectral accuracy of spectroradiometric devices is $4 \mathrm{~nm}$, monochromatic in this context means a spectral width of about that range. The full width at half maximum (FWHM) of the modes of the LCTF is between $7 \mathrm{~nm}$ and $15 \mathrm{~nm}$, depending upon the central wavelength chosen, and thus we can accept the assumption of monochromaticity. Nevertheless, if a more accurate result were needed, a bandwidth correction could be made ${ }^{32}$. Second, the radiometric information given by the CCD camera is accurate enough to guarantee that it does not depend upon the wavelength, the exposure time or other external factors. In the preceding sections we have described a precise procedure to assure this by means of a complete radiometric calibration.

As we said in Section 2, when using skylight as the source for spectral calibration, we must bear in mind that it is partially polarized. Hence the output of the LCTF will be affected by its orientation since it behaves as a linear polarizer. To avoid this problem, we use a linear polarizer with the same orientation as the LCTF to ensure that we always work with linearly polarized light and a fixed orientation for the polarizer elements. Figure 11 shows a schematic experimental setup used during this experiment. For each selected LCTF wavelength we take an image with the LCTF attached to the camera pointing to the zenith. In this way spatially homogeneous ${ }^{26}$ and monochromatic light (corresponding to the selected wavelength at the LCTF), $E_{2}$, impinges on the CCD array. Then we need to measure somehow the irradiance received by the camera in order to calculate its responsiveness at that wavelength. To this end, at the same time that the image is taken with the camera we measure the radiance from the zenith with the PR650 spectroradiometer, but with a linear polarizer (with the same orientation as the LCTF, i.e. $\theta_{1}=\theta_{2}$ ) attached to it in order to take into account only the amount of energy aligned with the transmission line of the LCTF. We have just measured $E_{1}$ (see Fig. 11). If we proceed in this way we only need to relate $E_{1}$ and $E_{2}$ to spectrally calibrate the camera, assuring that 
both radiances are linearly polarized with the same orientation $\left(\theta_{1}=\theta_{2}\right)$. To do this we first need to measure the spectral transmittance of the polarizer, $T_{p o l}(\lambda)$.

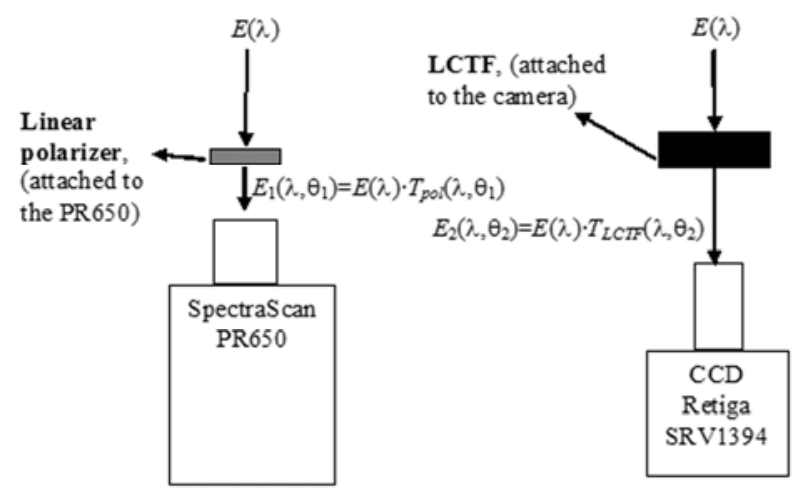

Figure 11. Schematic experimental setup for the spectral calibration of the CCD camera.

We use the light coming from a TFT monitor as linearly polarized, intense and covering the whole visible-range light source to measure the spectral transmittance of the polarizer. Using the PR650 as we explained in Section 2 we easily obtained the curve shown in Figure 12. Once we know $T_{p o l}(\lambda)$ and that $\theta_{1}=\theta_{2}$, we can relate $E_{1}$ and $E_{2}$ by looking at Figure 11 and calculating their ratio in order to eliminate their dependence upon $E(\lambda)$

$$
E_{2}(\lambda)=E_{1}(\lambda) \frac{T_{L C T F}(\lambda)}{T_{p o l}(\lambda)}
$$




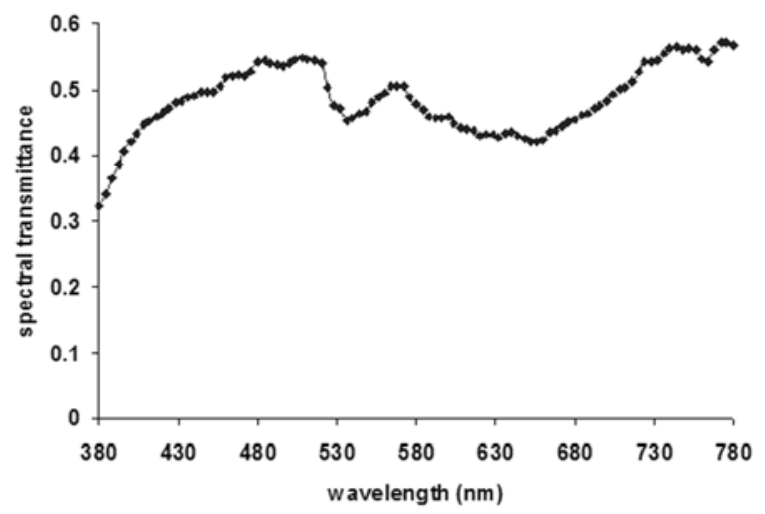

Figure 12. Spectral transmittance of the linear polarizer, $T_{p o l}(\lambda)$.

Once we know the irradiance impinging on the CCD camera at every wavelength we can easily relate its response to this incoming light, hence calculating its spectral responsiveness, which is shown in Figure 13 for the spatial average across the CCD matrix, also averaged over three series of measurements (the total standard deviation after the whole procedure is shown as error bars). In this same figure we also show the results of the spectral calibration performed by using three tunable lasers (two of which are Ar lasers and the other a dye laser) and an integrating sphere in the same configuration as that shown in Figure 3, which might cover the spectral regions from $454 \mathrm{~nm}$ to $514 \mathrm{~nm}$ and from $615 \mathrm{~nm}$ to $760 \mathrm{~nm}$. We can see how in the spectral regions where our method overlaps with the other method using tunable lasers the results are very similar, bearing in mind the difference in spectral bandwidth of both devices and the spectral feature of skylight, and also measurement uncertainty, which is about $4 \%$ for the PR650 and about $1.5 \%$ for the laser source method. Hence, we have proved that the LCTF can well be used as an accurate monochromator in calibration tasks, and also that skylight on perfectly clear days can be used as a very intense, spatially homogeneous, though partially polarized, light source. 


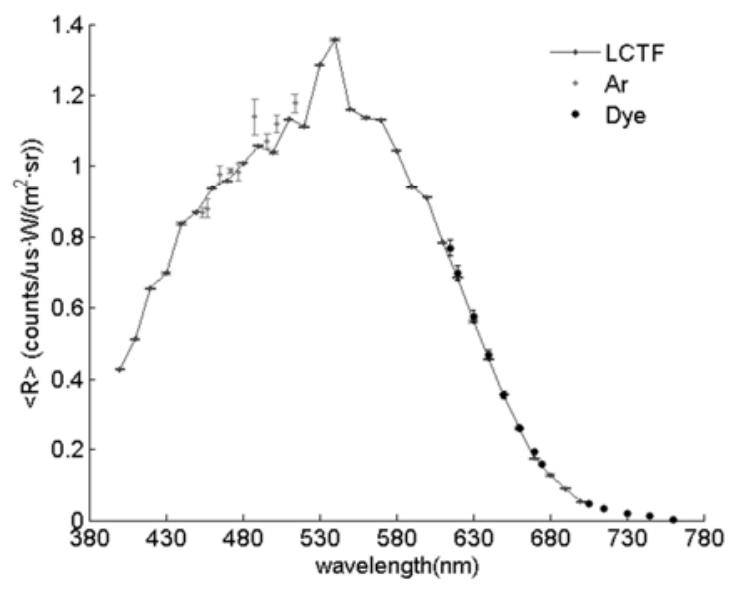

Figure 13. Spectral responsiveness of the CCD camera: Comparison of our method using the LCTF and a classical method using tunable lasers. Error bars correspond to the spatial standard deviation obtained across the whole CCD matrix and the three series of measurements.

\section{Spectroradiometric measurements using our calibrated multispectral system.}

In previous sections of this paper we have described the spectral calibration of the LCTF and the camera, and also corrected the camera's response to noise, non-linearity and spatial non-uniformity. Therefore we can immediately relate the corrected digital counts registered at a pixel of the camera, $C_{k}^{c}$, when a wavelength mode, $k$, is selected at the LCTF, with the incident irradiance at that wavelength, $E_{k}$.

$$
E_{k}=\frac{C_{k}^{c}}{R_{k} T_{L C T F, k} t_{\exp , k}}
$$

where $t_{\text {exp,k }}$ is the exposure time used for taking the image when mode $k$ was selected at the LCTF ( $T_{L C T F, k}$ being the spectral transmittance of this mode and $R_{k}$ the CCD responsiveness at this selected wavelength).

In Table 1 we show the mean values ( \pm standard deviations) for the various quality metrics explained below to compare the similarity between each pair of simultaneous spectra measured with the PR650 and our multispectral system over a set of 125 spectral measurements taken in Granada in 
2007 on the basis of Equation (10). Since the test set was acquired with the PR650 spectroradiometer between $380 \mathrm{~nm}$ and $780 \mathrm{~nm}$ at steps of $4 \mathrm{~nm}$, and the multispectral system gets spectral information between $400 \mathrm{~nm}$ and $720 \mathrm{~nm}$ every $10 \mathrm{~nm}$, a conversion of the data from the PR650 was made prior to comparing the spectra from both instruments. Hence we discarded the data below $400 \mathrm{~nm}$ and above $720 \mathrm{~nm}$ and made a linear interpolation in order to get spectral data every $10 \mathrm{~nm}$ (some intermediate data were also discarded). The metrics shown ${ }^{2,3}$ are the goodness fit coefficient (GFC), which is the cosine of the angle between two spectra if these are intended to be vectors in a Hilbert space, the colorimetric CIELAB $\Delta E_{a b}^{*}$ distance, the percentage of the integrated radiance error metric [IRE(\%)], which is a relative measurement of the difference in the total energy of the two spectral curves being compared, and the colorimetric and spectral combined metric (CSCM) proposed $^{2}$ to compare the spectra of natural illuminants from the colorimetric and spectral points of view, a measurement which has also been used by other researchers ${ }^{33}$. The equations defining these four metrics are shown below $\left[E(\lambda)\right.$ represents the original spectrum while $E_{R}(\lambda)$ stands for the recovered spectrum].

$$
\begin{gathered}
G F C=\frac{\left|\sum_{j} E\left(\lambda_{j}\right) E_{R}\left(\lambda_{j}\right)\right|}{\left|\sum_{j}\left[E\left(\lambda_{j}\right)\right]^{2}\right|^{1 / 2}\left|\sum_{j}\left[E_{R}\left(\lambda_{j}\right)\right]^{2}\right|^{1 / 2}} \\
\Delta E_{a b}^{*}=\sqrt{\Delta L^{* 2}+\Delta a^{* 2}+\Delta b^{* 2}} \\
\operatorname{IRE}(\%)=100 \frac{\left|\sum_{j=1}^{N} E\left(\lambda_{j}\right)-E_{R}\left(\lambda_{j}\right)\right|}{\sum_{j=1}^{N} E\left(\lambda_{j}\right)}
\end{gathered}
$$




$$
C S C M=\operatorname{Ln}(1+1000(1-G F C))+\Delta E_{a b}^{*}+\operatorname{IRE}(\%)
$$

Three examples of spectral reconstructions made by using this method, corresponding to the (a) $10^{\text {th }}$, (b) $50^{\text {th }}$, and (c) $90^{\text {th }}$ percentiles of the CSCM metric over the test set of 125 skylight measurements are shown in Figure 14, where it can be seen how the spectral measurements given by the multispectral system are quite similar to those given by the spectroradiometer PR650, although there is a tendency to overestimate the total energy of the spectra, which implies that high values are obtained for the IRE(\%) metric. This could be due to a systematic difference between the theoretically expected pixel values and the real ones, $C^{c}$, registered at the camera, which is brought about by the inexact assumption of monochromaticity of the LCTF transmittance modes. Nevertheless, the quality of the spectral measurements taken with the multispectral system may be accurate enough for certain purposes when studying skylight, where the total energy estimation is not of paramount importance and we may need only the relative SPD.

Table 1. Mean \pm standard deviation values of various metrics over the test set of 125 spectral measurements taken in Granada in 2007.

\begin{tabular}{cccc}
\hline GFC & ${\text { CIELAB } \Delta \boldsymbol{E}^{*}{ }_{\boldsymbol{a} \boldsymbol{b}}}$ & IRE (\%) & CSCM \\
\hline $0.998 \pm 0.002$ & $1.26 \pm 0.37$ & $13.1 \pm 7.1$ & $15.5 \pm 7.3$ \\
\hline
\end{tabular}




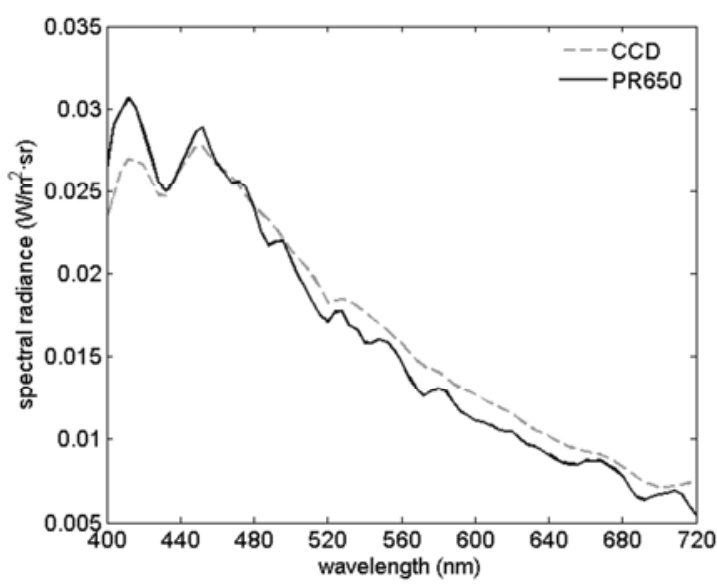

(a)

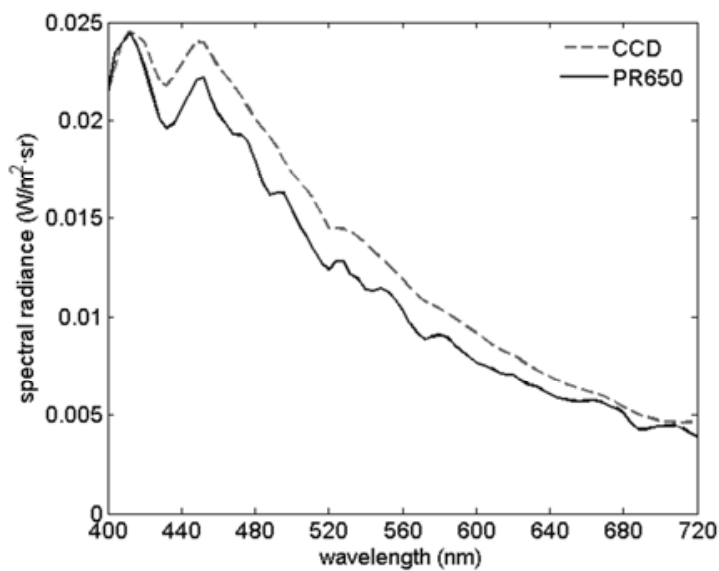

(b)

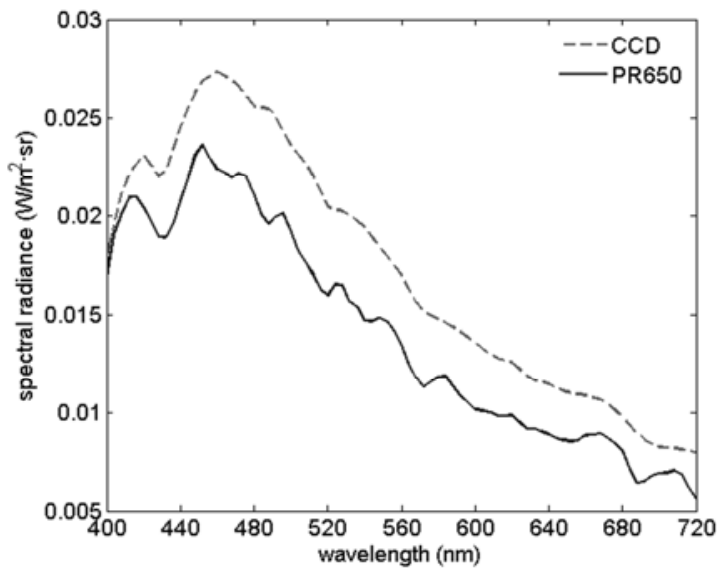

(c)

Figure 14. (a) $10^{\text {th }}$ percentile $(C S C M=6.00)$, (b) $50^{\text {th }}$ percentile $(C S C M=15.35)$ and (c) $90^{\text {th }}$ percentile $(\mathrm{CSCM}=25.05)$ over the test set of 125 spectral measurements taken in Granada in 2007. 


\section{Conclusions}

We propose here a way of calibrating the elements of a multispectral imaging system in such a way as to be able to use it as an accurate spectroradiometric instrument in a simple application for skylight imaging. By following the detailed steps described it is possible to eliminate the effects of some kinds of noise, correct the camera's response to non-linearity and non-uniformity and also to calculate the radiometric response of the CCD sensor array, leading to a substantial improvement in the quality of the CCD imaging.

The novelty of our method lies in our use of the LCTF in some parts of the calibration procedure as a simple monochromator and the zenith on perfectly clear days as a simple homogeneous object to be imaged. We have shown that an accurate estimation of the camera's spectral responsiveness can be obtained by using our approach and have compared the results against those obtained when using tunable lasers and integrating spheres under more controlled conditions.

We have shown a practical situation in which we use our calibrated multispectral system to obtain spectral measurements of skylight. Our results are very accurate when compared with measurements taken simultaneously with a PR650 spectroradiometer, demonstrating that the calibration procedure we present here is reliable for the purposes of spectral imaging.

\section{Acknowledgements}

This work was financed by the Spanish Red Temática "CIENCIA Y TECNOLOGÍA DEL COLOR" (FIS2005-25312-E), the Spanish Ministry of Education and Science, and the European Fund for Regional Development (FEDER) through grant FIS2007-60736. We thank our English colleague A. L. Tate for revising our English text. 


\section{REFERENCES}

1. J. Y. Hardeberg, Acquisition and reproduction of color images: colorimetric and multispectral approaches, (Dissertation.com, Parkland, Fla., 2001). (Revised second edition of Ph.D. dissertation, Ecole Nationale Supérieure des Télécommunications, Paris, 1999), pp. 121-174.

2. M. A. López-Álvarez, J. Hernández-Andrés, J. Romero, and R. L. Lee Jr., “Designing a practical system for spectral imaging of skylight”, Appl. Opt. 44, 5688-5695 (2005).

3. M. A. López-Álvarez, J. Hernández-Andrés, E. M. Valero and J. Romero, "Selecting algorithms, sensors and linear bases for optimum spectral recovery of skylight”, J. Opt. Soc. Am. A 24, 942956 (2007).

4. F. H. Imai and R. S. Berns, "Spectral estimation using trichromatic digital cameras”, in Proceedings of the International Symposium on Multispectral Imaging and Color Reproduction for Digital Archives (Society of Multispectral Imaging of Japan, Chiba, Japan, 1999), pp. 42-48.

5. H. Haneishi, T. Hasegawa, A. Hosoi, Y. Yokoyama, N. Tsumura, and Y. Miyake, "System design for accurately estimating spectral reflectance of art paintings”, Appl. Opt. 39, 6621-6632 (2000).

6. N. Shimano, K. Terai, and M. Hironaga, "Recovery of spectral reflectances of objects being imaged by multispectral cameras”, J. Opt. Soc. Am. A, 24, 3211-3219 (2007).

7. K. Chandra and G. Healey, "Estimating visible through near-infrared spectral reflectance from a sensor radiance spectrum”, J. Opt. Soc. of Am. A, 21, 1825-1833 (2004).

8. D. C. Day, Filter selection for spectral estimation using a trichromatic camera, M. Sc. dissertation, Rochester Institute of Technology (2003), (http://www.art-si.org/PDFs/Acquisition/DCDayMSThesis03.pdf). 
9. M. A. López-Álvarez, J. Hernández-Andrés, J. Romero, F. J. Olmo, A. Cazorla, and L. AladosArboledas, “Using a trichromatic CCD camera for spectral skylight estimation”, Appl. Opt. 47, H31-H38 (2008).

10. J. L. Nieves, E. M. Valero, S. M. C. Nascimento, J. Hernández-Andrés, and J. Romero, “Multispectral synthesis of daylight using a commercial CCD camera”, Appl. Opt. 44, 5696-5703 (2005).

11. M. Vilaseca, J. Pujol, M Arjona, and M. de Lasarte, “Multispectral system for reflectance reconstruction in the near infrared region”, Appl. Opt. 45, 4241-4253 (2006).

12. M. A. López-Álvarez, J. Hernández-Andrés, and J. Romero, “Developing an optimum computerdesigned multispectral system comprising a monochrome CCD camera and a liquid-crystal tunable filter”, Appl. Opt. 47, 4381-4390 (2008).

13. M. A. López-Álvarez, J. Hernández-Andrés, and J. Romero, "Building an optimum computerdesigned multispectral system for skylight acquisition”, in Proceedings of the $4^{\text {th }}$ European Conference on Color in Graphics, Imaging, and Vision (CGIV08), (Society for Imaging Science and Technology, Terrassa-SPAIN, June 2008), pp. 613-616.

14. S. M. C. Nascimento, F. P. Ferreira, and D. H. Foster, "Statistics of spatial cone excitation ratios in natural scenes”, J. Opt. Soc. Am. A, 19, 1484-1490 (2002).

15. http://micro.magnet.fsu.edu/primer/java/filters/aotf/index.html

16. G. E. Healey and R. Kondepudy, "Radiometric CCD Camera Calibration and Noise Estimation”, IEEE Trans. Pattern Anal. Machine Intell. 16, 267-276 (1994).

17. A. Ferrero, J. Campos, and A. Pons, "Low-uncertainty absolute radiometric calibration of a CCD”, Metrología 43, S17-S21 (2006). 
18. A. Ferrero, J. Campos, and A. Pons, "Experimental assessment of relative temporal fluctuation of CCD pixels”, Eur. Phys. J. Appl. Phys. 33, 225-228 (2006).

19. A. Ferrero, J. Campos, and A. Pons, "Correction of photoresponse nonuniformity for matrix detectors based on prior compensation for their nonlinear behaviour”, Appl. Opt. 45, 2422-2427 (2006).

20. J. Campos, “Radiometric calibration of charge-coupled device video cameras”, Metrologia 37, 459$464(2000)$

21. N. Shimano, "Suppression of noise effects in color correction by spectral sensitivities of image sensors”, Opt. Rev. 9, 81-88 (2002).

22. M. de Lasarte, J. Pujol, M. Arjona, and M. Vilaseca, “Optimized algorithm for the spatial nonuniformity correction of an imaging system based on a charge-coupled device color camera”, Appl. Opt. 46, 167-174 (2007).

23. S. Franco, Design with operational amplifiers and analog integrated circuits, $3^{\text {rd }}$ ed. (McGraw-Hill, Boston), 311-346 (2002).

24. R. A. Yotter and D. M. Wilson, "A review of photodetectors for sensing light-emitting reporters in biological systems”, IEEE Sens. J., 3, 288-303 (2003).

25. P. L. M. Geladi, “Calibration Standards and Image Calibration” in Techniques and Applications of Hyperspectral Image Analysis, $1^{\text {st }}$ ed (John Wiley and sons), 203-220 (2007).

26. J. Hernández-Andrés, R. L. Lee, and J. Romero, “Color and luminance asymetries in the clear sky”, Appl. Opt. 42, 458-464 (2003).

27. http://www.cri-inc.com/support/components.asp

28. D. H. Foster, S. M. C. Nascimento, and K. Amano, "Information limits on neural identification of colored surfaces in natural scenes”, Vis. Neurosci., 21, 331-336 (2004). 
29. J. R. Janesick, Scientific Charge-Coupled Devices, SPIE Press (Bellingham, Washington), Chap. 2, 6 and 7 (2001).

30. http://www.qimaging.com/products/datasheets/Retiga-SRV.pdf

31. A. Alsam and R. Lenz, “Calibrating color cameras using metameric blacks”, J. Opt. Soc. Am. A, 24, 11-17 (2007).

32. J. Campos, A. Corrons, A. Pons, P. Corredera, J. L. Fontecha and J. R. Jiménez, "Spectral responsivity uncertainty of silicon photodiodes due to calibration spectral bandwith”, Meas. Sci. Technol. 12, 1926-1931 (2001).

33. P. A. García, R. Huertas, M. Melgosa, and G. Cui, "Measurement of the relationship between perceived and computed color differences”, J. Opt. Soc. Am. A 24, 1823-1829 (2007). 